\title{
3D Arbitrary Channel Fabrication for Lab on a Chip Applications using Chemical Decomposition
}

\author{
Jahan Zeb Gul ${ }^{1}$, Jinhee $\mathrm{Na}^{2}$, ${ }^{*}$ Kyung Hyun $\mathrm{Choi}^{1}$ \\ ${ }^{1}$ Department of Mechatronics Engineering, \\ ${ }^{1}$ Jeju National University, Jeju 690-756, South Korea \\ ${ }^{2}$ Biophilic Ltd, 152, Juggunro, Youngin-si, Gyunggi-do, South Korea.
}

\begin{abstract}
This article demonstrate a simple method to use of three-dimensionally (3D) printed molds that are chemically decomposable for rapid fabrication of complex and arbitrary microchannel geometries. These complex microchannel are unachievable through existing soft lithography techniques. The molds are printed directly from hand held $3 D$ printing pen that can print in midair, making rapid prototyping of microfluidic devices possible in hours. PLA based copper filament is used to print the arbitrary channels. The printed channels are then placed inside PDMS and PDMS is cured. The cured sample is then immersed in chemical solution (Acetic Acid + Sodium Chloride+ Hydrogen peroxide), which decomposes the PLA based copper channel thus leaving an empty channel inside the PDMS block. This method enable precise control of various device geometries, such as the profile of the channel cross-section and variable channel diameters in a single device.
\end{abstract}

Keywords- Micro Channel, Arbitrary, 3D Micro Channel, Lab on a Chip.

\section{INTRODUCTION}

Microfluidics is anunceasinglydeveloping field of great importance in drug discovery[1], physics[2], [3], biology[4], [5], biomedical research[6], and organs-on-chip[7]. The slightvolumes of liquid required fortrials, the behavior of fluids at the micro domain and the lab-on-chip method www.ijeab.com make microfluidics one of the interdisciplinary field par excellence[8]. For the fabrication of microfluidic devices, polydimethylsiloxane (PDMS) is the most general material in research laboratories because of its low cost, gas permeable and has refractive index of 1.4 (transparent)[9]-[15]. Conventional fabrication strategies for the fabrication of microfluidic micro channels are essentially two-dimensional (2D) which confine the geometries of micro channels within 2D planes. A master is designed first which is usually obtained by clean-room lithography of silicon wafers. PDMS is poured on the master, and after curing, the rubber is peeled off from the master and subsequently chemically bonded to another surface after activation with oxygen plasma or using chemical solutions. The main limitations with PDMS is that, the fabrication method of PDMS is considered too complex for many junior scientists without any experience in microfabrication[16]. There are a lot of alignment issues when multiple layers of PDMS are stacked and sealed using oxygen plasma treatment[17].

The ability to make 3D micro channels is complex and standard fabrication method cannot achieve precise and accurate results. 3D micro channels also adds functionality in devices such as micro valves and mixers. Another benefit of $3 \mathrm{D}$ structures is that it can increase the areal density of micro-components by vertical stacking, to replicate the complex microvasculature found in living creatures, and

Page | 2340 
ease integration of electronics and optics.

Recently there has been progress towards employing 3Dprinting to fabricate microfluidic devices of desired complexities[18]-[22]. This method involves the creation of 3D objects using layer by layer deposition approach. 3D printing is successfully employed in tissue engineering to develop scaffolds based on hard polymericmaterials and hydrogels[23].However such techniques require the use of manual assembly for producing large scale structures. Thus the fabrication of 3D microfluidic channels with defined microarchitectural details in cost-effective, scalable manner remains a challenge. Another method involves, sacrificial mold or fugitive ink for the fabrication of PDMS microfluidic devices[18], [24]-[26]. Although the use of sacrificial mold is a step forward in simplifying the fabrication of microfluidic devices, it still requires either harsh condition like the use of high temperatures for creatingor removing, a template, applying heavy swelling for pulling out the template, or the use of complex mold fabrication.

Taken in sum, most fabrication processes generally involves 2D micro channels[27]-[30], but with some effort, can form $3 \mathrm{D}$ channels by bonding multiple layers. Bonding involves the need for challenging alignment of layers at micro-scale.Each of these fabrication technique adds additional steps, time, expertise and cost in the fabrication process. In addition, the bonded interface is typically a weak-point in the device that is prone to leakage. Moreover, embedding other functionalities such as sensors, valves and mixers is extremely hard or even impossible using these methods.

Here we present an easy two-step chemically decomposablePLA based copper filament removal method for achieving arbitrary 3Dmicrometric channels in a single block of PDMS. The PLA based copper filament is 3D printable. A microchannel structure is first 3D printed and then immersed in PDMS block and cured. The cured PDMS is then placed inside an organic solution of acetic acid, sodium chloride and hydrogen peroxide. The organic solution decompose the PLA based copper structure inside the PDMS block thus leaving a hollow 3D channel. The channel is then washed with water to remove impurities.Using the chemically scaffold-removal method, there is no need of lithography steps nor silicon masters, no need of bonding the PDMS on surfaces nor of repetitive procedures for obtaining multi- level channels, making the fabrication of microfluidic devices easy, low-cost and opening up the field for a plethora of scientists working in different areas.

\section{EXPERIMENTAL}

An easy two-step chemically decomposable 3D printed PLA based copper structure-removal method for achieving 3Dmicrometric channels in a single block of PDMS. Using the thiscopper removal method, there is no need of lithography steps nor silicon masters, no need of bonding the PDMS on surfaces nor of repetitive procedures for obtaining multi-level channels, making the fabrication of microfluidic devices easy, low-cost and opening up the field for a plethora of scientists working in different areas. 


a \begin{tabular}{|c|l|l|}
\hline S.No. & \multicolumn{1}{|c|}{ Property } & \multicolumn{1}{|c|}{ PDMS } \\
\hline 1. & Mechanical behavior & Elastomeric \\
\hline 2. & Thermal range & $\sim 80^{\circ} \mathrm{C}$ \\
\hline 3. & Acid/base resistance & Not Good \\
\hline 4. & Solvent resistance & Not Good \\
\hline 5. & Optical transmissivitv (visible range) & Excellent \\
\hline 6. & Biocompatibilitv & Biocompatible \\
\hline
\end{tabular}

b

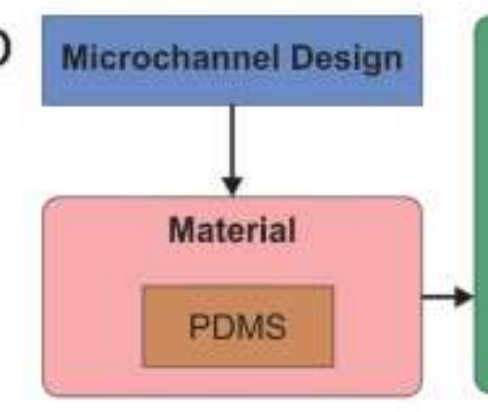

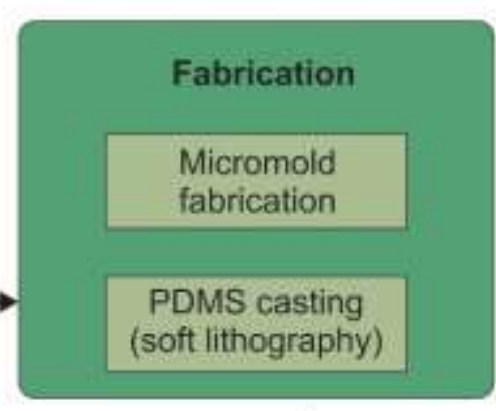

\section{Fabrication}

Micromold

fabrication

PDMS casting

(soft lithography)
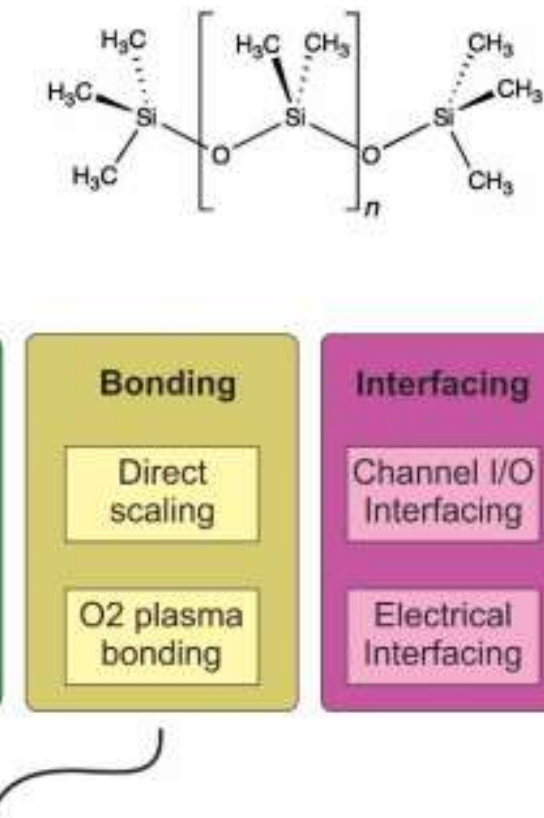

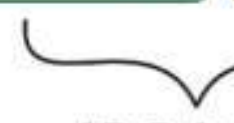

3D Printing

Fig, 1(a) Properties of PDMS with its molecule (b) Illustration of microchannel design process with PDMS. The conventional casting and bonding steps are replaced with $3 D$ printing.

PDMS is widely used material because of various properties shown in Fig.1(a). The chemical decomposition method replaces the conventional fabrication of microchannel using PDMS. The two steps i.e. molding and bonding is replaced with $3 \mathrm{D}$ printing here as shown in Fig. 1(b). The material used here is commercially available $3 \mathrm{D}$ printable PLA based copper filament. The technique is inspired by etching technique of printed circuit boards. The copper is decomposed in printed circuit board etching using acidic chemicals such as ferric chloride. Here in order to save the PDMS shape and microchannel architecture, a less sensitive organic compound of acetic acid, sodium chloride and hydro peroxide is used to decompose the copper. The decomposition time is long as compared to original decomposition chemical i.e. ferric chloride but there is no or negligible harm with organic solution. 
a Printable Copper $+3 \mathrm{D}$ Printing Pen
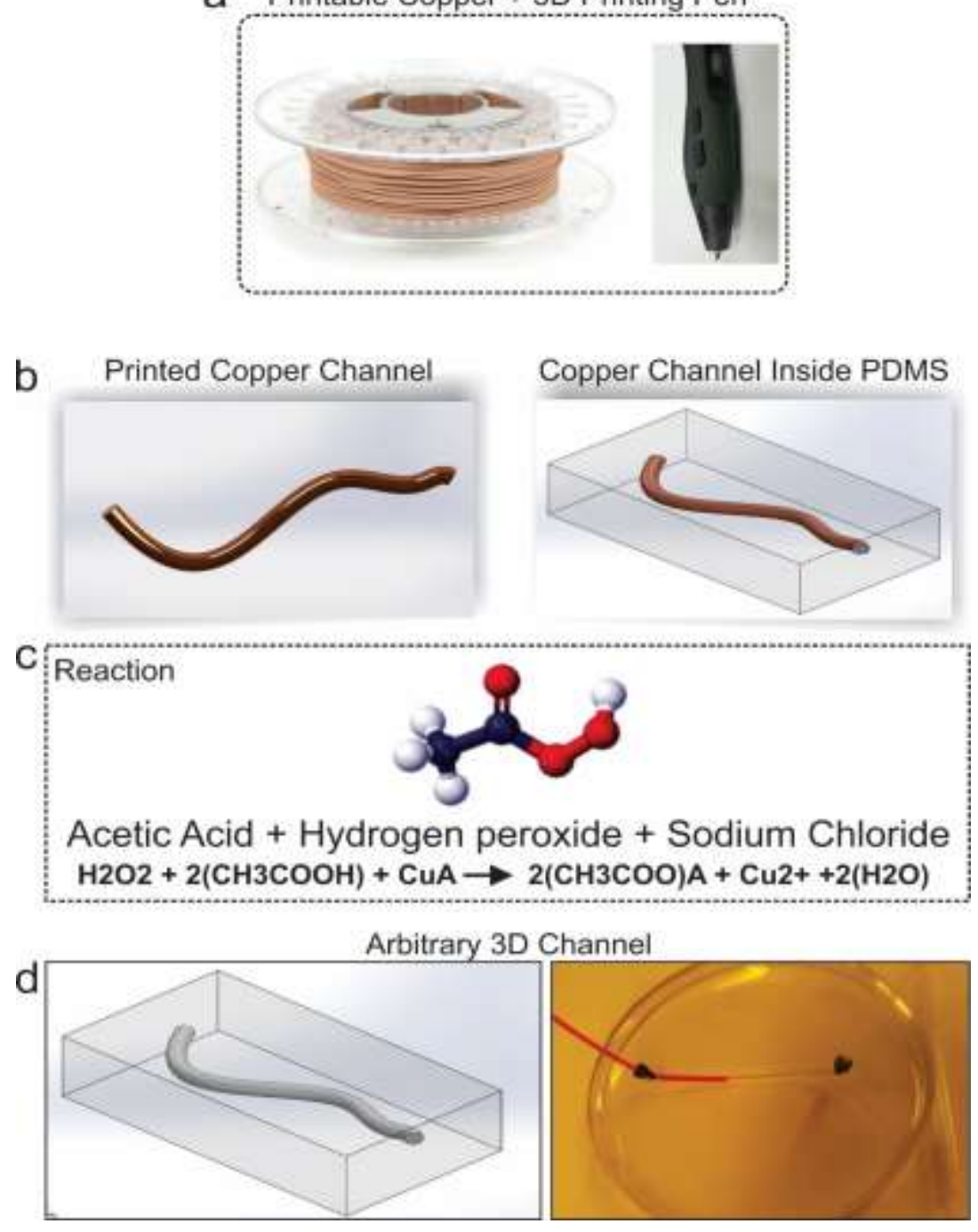

Fig.2: Illustration of micro channel fabrication using chemically decomposable and 3D printed PLA based copper. (a) The channel shape is $3 D$ printed using hand held $3 D$ pen. (b) An arbitrary $3 D$ channel of copper filament. The copper channel is then set inside PDMS mold and PDMS is cured. (c) The PDMS mold is then immersed in organic solution of acetic acid, sodium chloride and hydrogen peroxide. (d) The decomposition of copper inside the PDMS results in a hollow channel inside PDMS. The actual photograph of fabricated channel is also shown.

\section{RESULTS AND DISCUSSION}

A cross-section of an emptied microchannel has an exact circular profile. The circular profile arises from the shape because of midair 3D printing of PLA based copper using a hand held 3D printing pen which consists of a conical polypropylene needle. It is possible to control the width of the features simply by varying the diameter of the nozzle and that the line widths are uniform (e.g. a needle with a $200 \mu \mathrm{m}$ diameter produces lines with a standard deviation www.ijeab.com of $200 \mu \mathrm{m})$. If 3D printing of copper filament is done on a substrate or a FDM printer with stage, the cross section of an emptied micro channel in this case will be in semi-circular profile. The semi-circular profile is because of natural adoption of copper metal on straight surface. In this case, it is also possible to control the width by simply changing the diameter of the nozzle e.g. a nozzle with a $200 \mu \mathrm{m}$ diameter produces lines with a standard deviation of $7 \mu \mathrm{m}$. The printed structure is the then cured inside PDMS 
using acetic acid, hydrogen peroxide and sodium chloride.

The chemical reaction and materials are shown in Fig.2(a)(c).

Fig. 3 shows the images of the fabricated micro channels inside PDMS using organic decomposition of PLA based copper. Two types of channels are fabricated. One a simple straight bridge shaped channel with single inlet/outlet and second is a complex helical structure with single inlet/outlet. The diameter of the both channels are 200 and $250 \mu \mathrm{m}$ respectively. The length of the bridge channel and helical channels are $6 \mathrm{~cm}$ and $4 \mathrm{~cm}$ respectively. A red color liquid is flown through these channels to verify the smooth flow. The SEM images shown in Fig. 3(b) is the cross sectional images of both channels. The images indicates a smooth channel with no or negligible harm to PDMS. A two inlet and one outlet helical mixer channel is then fabricated to proves the potential of various embedded concepts using the chemical decomposition method. The fabricated mixer channel shown in Fig. 3(c) is smooth and can mix two liquids if certain parameters are kept in control such as velocity of incoming liquids or viscosity of liquids etc.

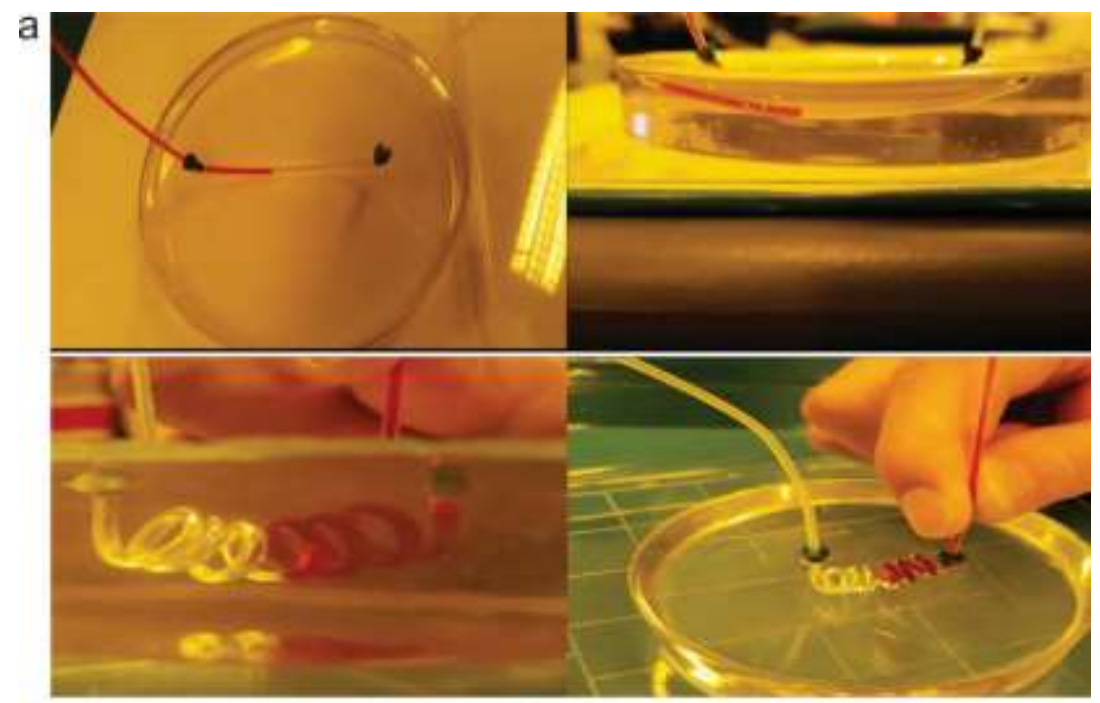

b

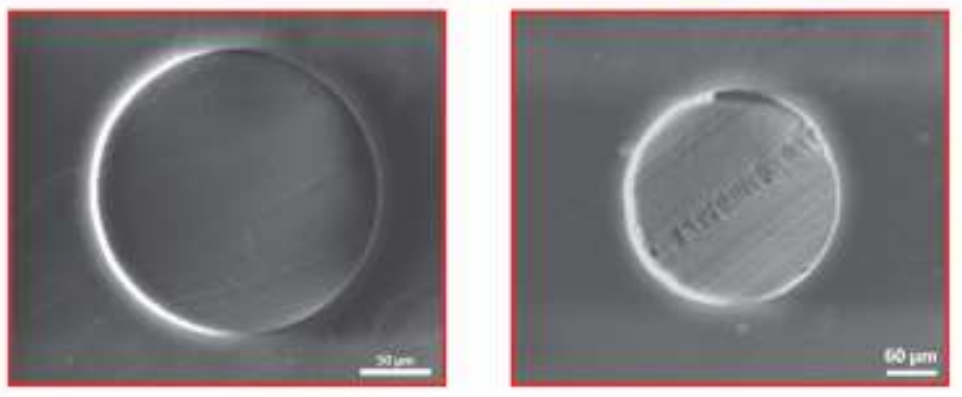

C

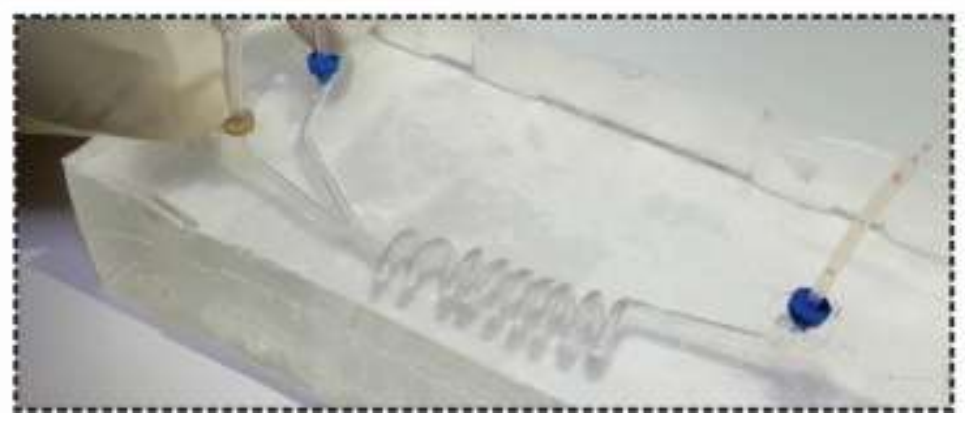

Fig.3 (a)Images of the fabricated micro channels inside PDMS using organic decomposition of PLA based copper. An arbitrary hanging bridge channel and a helical channel. (b) SEM Images of the cross section of a straight and helical channels (d) A two inlet one outlet helical mixer channel. 
Fig.4 shows results of the cost comparison and copper decomposition duration between the conventional copper etching material Ferric Chloride and Organic Compound (Acetic Acid, Sodium Chloride, and Hydrogen peroxide). The results shown in Fig.4 proves that conventional material i.e. Ferric Chloride can decompose the copper
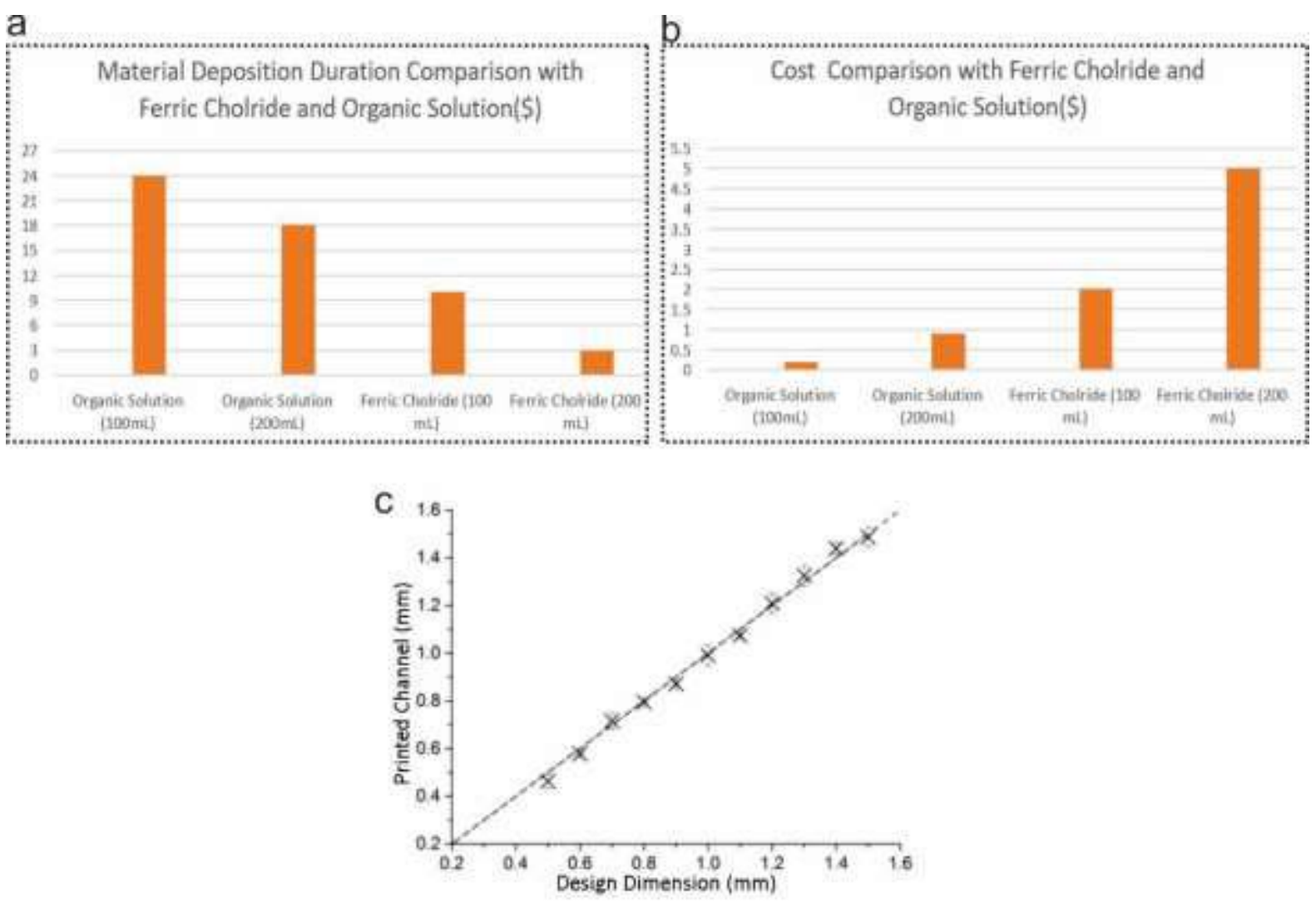

Fig.4: Results of time comparison and cost comparison with ferric chloride (a conventional etching material) with organic compound (acetic acid, hydrogen peroxide and sodium chloride). (a) Duration comparison results (b) Cost comparison results (c) The linear behavior of the fabricated channel w.r.t. model in software proves the efficiency and accuracy of

\section{fabrication method.}

The consistency of dimensions of the fabricated microchannels in all directions, is shown in Fig. 4(c), although there is some unavoidable roughness resulting from the layer by layer nature of copper PLA printing as can be seen in Fig $3 b$ however, the channel surface roughness was not considered for optimization in this work.

\section{CONCLUSION}

Chemically decomposable 3D printed structures are used for the fabrication of PDMS channels demonstrating www.ijeab.com variable channel dimensions as small as $100 \mathrm{~s}$ of micrometers in all-PDMS channels. The fabricated 3D arbitrary micro channel is expected to exactly mimic the human blood channels. These channels will be used in various applications such as LOC platforms. The fabrication of 3D arbitrary channels and investigation of fluidic motion inside them has laid a new foundation with promising applications in the area specially related to LOC, artificial organs, bio medical applications and implantable devices. 


\section{CONFLICT OF INTEREST}

The authors declare that they have no conflict of interest.

\section{AUTHOR CONTRIBUTIONS}

J.Z.G. performed the experiments and also wrote the paper. The analysis of the results and the final revision of the paper was done by J.N and K.H.C.

\section{REFERENCES}

[1] P. Neuži, S. Giselbrecht, K. Länge, T. J. Huang, and A. Manz, "Revisiting lab-on-a-chip technology for drug discovery," Nat. Rev. Drug Discov., vol. 11, no. 8, pp. 620-632, Aug. 2012.

[2] H. A. Stone and S. Thutupalli, "Microfluidics: For a few drops more," Nat. Phys., vol. 10, no. 2, pp. 87-88, Jan. 2014.

[3] L. Vinet and A. Zhedanov, "A 'missing' family of classical orthogonal polynomials," Rev. Mod. Phys., vol. 77, no. 3, pp. 977-1026, Nov. 2010.

[4] D. J. Beebe, G. a Mensing, and G. M. Walker, "Physics and Applications of Microfluidics in Biology," Annu. Rev. Biomed. Eng., vol. 4, no. 1, pp. 261-286, Aug. 2002.

[5] S. K. Sia and G. M. Whitesides, "Microfluidic devices fabricated in Poly(dimethylsiloxane) for biological studies," Electrophoresis, vol. 24, no. 21, pp. 3563-3576, Nov. 2003.

[6] E. K. Sackmann, A. L. Fulton, and D. J. Beebe, "The present and future role of microfluidics in biomedical research," Nature, vol. 507, no. 7491, pp. 181-189, Mar. 2014.

[7] S. N. Bhatia and D. E. Ingber, "Microfluidic organs-on-chips," Nat. Biotechnol., vol. 32, no. 8, pp. 760-772, Aug. 2014.

[8] D. Mark, S. Haeberle, G. Roth, F. von Stetten, and R. Zengerle, "Microfluidic lab-on-a-chip platforms: requirements, characteristics and applications," Chem. Soc. Rev., vol. 39, no. 3, p. 1153, 2010.

[9] S. K. W. Dertinger, D. T. Chiu, N. L. Jeon, and G. M. www.ijeab.com
Whitesides, "Generation of Gradients Having Complex Shapes Using Microfluidic Networks," Anal. Chem., vol. 73, no. 6, pp. 1240-1246, Mar. 2001.

[10] K. Ren, Y. Chen, and H. Wu, "New materials for microfluidics in biology," Curr. Opin. Biotechnol., vol. 25, pp. 78-85, Feb. 2014.

[11] K. Ren, J. Zhou, and H. Wu, "Materials for Microfluidic Chip Fabrication," Acc. Chem. Res., vol. 46, no. 11, pp. 2396-2406, Nov. 2013.

[12] C.-W. Tsao, "Polymer Microfluidics: Simple, Low-Cost Fabrication Process Bridging Academic Lab Research to Commercialized Production," Micromachines, vol. 7, no. 12, p. 225, Dec. 2016.

[13] A. Alrifaiy, O. A. Lindahl, and K. Ramser, "Polymer-Based Microfluidic Devices for Pharmacy, Biology and Tissue Engineering," Polymers (Basel)., vol. 4, no. 4, pp. 1349-1398, Jul. 2012.

[14] M. Figurova, D. Pudis, P. Gaso, and I. Cimrak, "PDMS microfluidic structures for LOC applications," in 2016 ELEKTRO, 2016, pp. 608-611.

[15] J. Friend and L. Yeo, "Fabrication of microfluidic devices using polydimethylsiloxane," Biomicrofluidics, vol. 4, no. 2, p. 26502, Jun. 2010.

[16] Q. Zhang and R. H. Austin, "Applications of Microfluidics in Stem Cell Biology," Bionanoscience, vol. 2, no. 4, pp. 277-286, Dec. 2012.

[17] H. Wu, T. W. Odom, D. T. Chiu, and G. M. Whitesides, "Fabrication of Complex Three-Dimensional Microchannel Systems in PDMS," $J$. Am. Chem. Soc., vol. 125, no. 2, pp. 554-559, Jan. 2003.

[18] D. P. Parekh, C. Ladd, L. Panich, K. Moussa, and M. D. Dickey, "3D printing of liquid metals as fugitive inks for fabrication of 3D microfluidic channels," Lab Chip, vol. 16, no. 10, pp. 1812-1820, 2016.

[19] C. Chen, B. T. Mehl, A. S. Munshi, A. D. Townsend, D. M. Spence, and R. S. Martin, "3D-printed microfluidic devices: fabrication, advantages and limitations - a mini review," Anal. Methods, vol. 8, no. 
31, pp. 6005-6012, 2016.

[20] G. Gaal, M. Mendes, T. P. De Almeida, M. H. O.

Piazzetta, Â. L. Gobbi, A. Riul, and V. Rodrigues, "Sensors and Actuators B: Chemical Simplified fabrication of integrated microfluidic devices using fused deposition modeling 3D printing," Sensors Actuators B. Chem., vol. 242, pp. 35-40, 2017.

[21] G. Gaal, M. Mendes, T. P. de Almeida, M. H. O. Piazzetta, Â. L. Gobbi, A. Riul, and V. Rodrigues, "Simplified fabrication of integrated microfluidic devices using fused deposition modeling 3D printing," Sensors Actuators B Chem., vol. 242, pp. 35-40, Apr. 2017.

[22] S. Waheed, J. M. Cabot, N. P. Macdonald, T. Lewis, R. M. Guijt, B. Paull, and M. C. Breadmore, "3D printed microfluidic devices: enablers and barriers," Lab Chip, vol. 16, no. 11, pp. 1993-2013, 2016.

[23] S. Mohanty, L. B. Larsen, J. Trifol, P. Szabo, H. V. R. Burri, C. Canali, M. Dufva, J. Emnéus, and A. Wolff, "Fabrication of scalable and structured tissue engineering scaffolds using water dissolvable sacrificial 3D printed moulds," Mater. Sci. Eng. C, vol. 55, pp. 569-578, Oct. 2015.

[24] J. Hammer, L.-H. Han, X. Tong, and F. Yang, "A Facile Method to Fabricate Hydrogels with Microchannel-Like Porosity for Tissue Engineering," Tissue Eng. Part C Methods, vol. 20, no. 2, pp. 169-176, Feb. 2014.
[25] D. J. Beebe, J. S. Moore, J. M. Bauer, Q. Yu, R. H Liu, C. Devadoss, and B.-H. Jo, "Functional hydrogel structures for autonomous flow control inside microfluidic channels : Abstract: Nature," Nature, vol 404, no. 6778, pp. 588-590, 2000

[26] S. Li, Y. Liu, Y. Li, C. Liu, Y. Sun, and Q. Hu, “A novel method for fabricating engineered structures with branched micro-channel using hollow hydrogel fibers," Biomicrofluidics, vol. 10, no. 6, p. 64104, Nov. 2016.

[27] M. D. Raj and R. Rengaswamy, "Investigating Arrangement of Composite Drops in Two-Dimensional Microchannels Using Multiagent Simulations: A Design Perspective," Ind. Eng. Chem. Res., vol. 54, no. 43, pp. 10835-10842, Nov. 2015.

[28] M. J. A. Khan, M. R. Hasan, and M. A. H. Mamun, "Flow Behavior and Temperature Distribution in Micro-Channels for Constant Wall Heat Flux," Procedia Eng., vol. 56, pp. 350-356, 2013.

[29] G. Puccetti, B. Pulvirenti, and G. L. Morini, "Experimental Determination of the 2D Velocity Laminar Profile in Glass Microchannels using $\mu$ PIV," Energy Procedia, vol. 45, pp. 538-547, 2014.

[30] X. Guo, C. Huang, A. A. Alexeenko, and J. P. Sullivan, "Numerical and Experimental Study of Gas Flows in 2D and 3D Microchannels," in ASME 5th International Conference on Nanochannels, Microchannels, and Minichannels, 2007, pp. 393-400. 